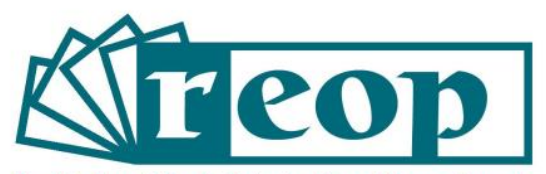

Revista Española de Orientación y Psicopedagogía

\title{
VALIDACIÓN DE UN INSTRUMENTO PARA DETECTAR NECESIDADES DE ORIENTACIÓN EN ALUMNADO UNIVERSITARIO DE NUEVO INGRESO ${ }^{1}$
}

\section{VALIDATION OF AN INSTRUMENT TO DETECT GUIDANCE NEEDS IN NEW UNIVERSITY STUDENTS}

\author{
Manuel Delgado-García ${ }^{2}$ \\ Universidad de Huelva. Facultad de Educación, Psicología y Ciencias del Deporte. Departamento \\ de Pedagogía. Huelva, España \\ Sara Conde Vélez \\ Universidad de Huelva. Facultad de Educación, Psicología y Ciencias del Deporte. Departamento \\ de Pedagogía. Huelva, España \\ Mํㅡㄹ Carmen Azaustre Lorenzo \\ Universidad de Huelva. Facultad de Educación, Psicología y Ciencias del Deporte. Departamento \\ de Pedagogía. Huelva, España
}

\section{RESUMEN}

El ingreso en la Universidad trasciende a los estudiantes desde múltiples dimensiones (organizativas, sociales, personales) y su adaptación va a depender de sus destrezas para afrontar y superar las necesidades que surjan. Esta investigación tiene como objetivo diseñar y someter a procesos de validación un instrumento para conocer la incidencia e interacción de determinados factores (ligados a motivaciones personales $u$ orientaciones académicas y hacia la carrera) en la generación de esas necesidades. Se utiliza una metodología tipo encuesta para distribuir un cuestionario entre los estudiantes del primer curso de diferentes grados de la Facultad

\footnotetext{
1 Financiación: Ayudas a la innovación docente e investigación educativa para la mejora de la docencia en la Universidad de Huelva para el curso 2018/2019. Código PIE005.

${ }^{2}$ Correspondencia: Manuel Delgado-García. Correo-e: manuel.delgado@dedu.uhu.es
} 
de Educación, Psicología y Ciencias del Deporte de la Universidad de Huelva. El instrumento está constituido por 3 escalas (motivaciones de ingreso; necesidades de orientación académica; necesidades de orientación para la carrera) que incorporan indicadores clave para comprender la realidad del primer contacto del alumno con sus estudios universitarios. Los resultados ofrecen análisis descriptivos y de correlación de cada una de estas escalas para acceder a las interacciones entre los factores emergentes; también se establecen procedimientos de validación y fiabilidad del instrumento mediante la valoración de la consistencia interna y el análisis factorial de cada escala. Como conclusión, se ofrece un instrumento útil para orientadores y profesorado universitario, con rigurosas propiedades psicométricas que dejan entrever la existencia de siete factores clave para ayudar a comprender el constructo teórico de referencia y de esta forma contribuir a la detección y evaluación de necesidades de orientación en los estudiantes de nuevo ingreso en la universidad.

Palabras clave: Intervención; evaluación de necesidades; orientación; enseñanza superior; validación de instrumentos.

\begin{abstract}
Admission to the University transcends students from multiple dimensions (organizational, social, personal) that they usually face alone, and their adaptation will depend largely on their skills to meet and overcome the needs that arise. This research aims to design and subject to validation processes an instrument to know the incidence and interaction of certain factors (linked to personal motivations or academic and career orientations) in the generation of these needs. A transversal research design is used based on a survey methodology to distribute an instrument among the students of the first year of different degrees of the Faculty of Education, Psychology and Sports Sciences of the University of Huelva. The instrument consists of 3 scales (profile-motivations for admission; academic orientation needs; career guidance needs) that incorporate key indicators to understand the reality of the student's first contact with their university studies. The results offer descriptive and correlational analyses of each of these scales to access the interactions between the emerging factors. Validation and reliability procedures of the instrument are also established by assessing the internal consistency and factor analysis of each scale. In conclusion, it offers a useful instrument for counselors and university professors, with rigorous psychometric properties that reveal the existence of seven key factors to help understand the theoretical construct of reference and thus contribute to the detection and evaluation of guidance needs in new university students.
\end{abstract}

Key Words: Intervention; needs assessment; guidance; higher education; test validity.

\title{
Cómo citar este artículo:
}

Delgado-García, M., Conde Vélez, S. y Azaustre Lorenzo, M.C. (2021). Validación de un instrumento para detectar necesidades de orientación en alumnado universitario de nuevo ingreso. Revista Española de Orientación y Psicopedagogía, 32(1), 92-115. https://doi.org/10.5944/reop.vol.32.num.1.2021.30742 


\section{Introducción}

En España la orientación educativa, incluida la etapa universitaria, se consolida en la década de los 90 y primera del siglo XXI, momento en que se desarrollan numerosos estudios que justifican la presencia y la necesidad de abordar la orientación en el ámbito universitario (Campoy y Pantoja, 2000; Rodríguez-Moreno, 2002; Fernández-Díaz, 2006), pues se entiende que es un área prioritaria para la atención hacia los estudiantes y desde la que se pretende ir más allá de los límites del aula (Domingo et al., 2016) y favorecer su adaptación a la universidad, su rendimiento y su persistencia en el sistema (Figuera et al., 2015).

Álvarez-Pérez y López-Aguilar (2019) consideran que la orientación en la universidad española es un elemento necesario para ayudar al alumnado en múltiples aspectos y también es un elemento de calidad que enriquece el proceso de enseñanza-aprendizaje; sin embargo, Suárez Lantarón (2014) y Muñoz y Gairín (2013) destacan la presencia de ciertas limitaciones que acaban por condicionar esta finalidad: a) la existencia de una realidad heterogénea relativa a la tipología de servicios y acciones; b) la dificultad para la integración y coordinación en las intervenciones puntuales e informativas y c) la carencia de recursos materiales y humanos para desarrollar los fundamentos de la acción orientadora. Atendiendo a esta realidad, este estudio profundiza en la detección de necesidades de orientación en estudiantes universitarios de nuevo ingreso, abordando diferentes variables (motivaciones personales hacia los estudios, las necesidades ligadas al plano académico o las próximas al acompañamiento durante dichos estudios) que la literatura destaca como claves en su proceso de adaptación y consolidación (Figuera y González, 2014; Hofstadt et al., 2005; Manzano-Soto y Roldan-Morales, 2015; Sánchez-García et al., 2011; Moreno-Yaguana y Sánchez-García, 2018; Pereira et al., 2019).

En primer término, el perfil motivacional de ingreso de los estudiantes universitarios es analizado desde diferentes modelos teóricos, de entre los cuales destacan el modelo psicológico, el modelo de adaptación, los modelos económicos, el modelo organizacional y el modelo psicopedagógico (Íñiguez et al., 2016). Los primeros modelos toman como punto de partida aquellas variables de índole psicoeducativas y con un carácter interno, como puede ser la ausencia de motivación en el estudiantado cuando inicia sus estudios (Belloc et al., 2011), la falta de capacidad a la hora de esperar recompensas o superar dificultades (Landry, 2003), o las barreras ligadas a la integración social y autopercepción que experimenta y a las que hace frente el alumnado (Harrison, 2018). Concretamente, el modelo psicológico destaca como factores la motivación (Cabrera et al., 2006), las expectativas y la percepción (Artís et al., 2008). Por su parte, el modelo de adaptación pone el énfasis en motivos socioacadémicos como pueden ser la satisfacción académica y la relación con el grupo de iguales que demuestra el estudiante (Escolano et al., 2019).

En segundo término, las investigaciones que han puesto el foco de atención en las necesidades de orientación universitaria comparten la idea que los Programas y Servicios de Orientación han de cumplir diversas funciones centradas en orientar e informar sobre aspectos académicos, profesionales, de inserción laboral, de prevención del abandono universitario o de atención a la diversidad (Farriols et al., 2014; Hernández et al., 2015; Llanes et al., 2017; Vergara et al., 2017), por lo que han favorecido la elaboración de estudios centrados en señalar aquellos factores asociados al éxito académico (Álvarez-Pérez y López-Aguilar, 2016) o bien dar a conocer los obstáculos del estudiantado en el acceso a la universidad (Urbanaviciute et al., 2016); en consecuencia, Moreno-Yaguana y Sánchez García (2018) afirman que "el diagnóstico de dichas necesidades debe entenderse como un proceso sistemático dirigido a identificar las carencias y dificultades, pero también las potencialidades percibidas" (p.97). 
Existen algunas necesidades específicas en el ámbito de la orientación académica como la de solicitar información sobre los perfiles de las carreras que eligen los estudiantes; la indagación sobre las características del modelo educativo de la Universidad relativo a las competencias: métodos y estrategias de estudio adecuados, estrategias de búsqueda de información y de metodologías de trabajo científico que conlleven a un aprendizaje autónomo y crítico; la orientación sobre los nuevos planes de estudio, con la finalidad de que los estudiantes sean capaces de asumir y resolver las situaciones de mayor complejidad como las referidas a las elecciones que debe realizar de forma continua sobre contenidos académicos específicos (Martínez, 2009); y también se contemplan otras necesidades propias del área de la orientación para la carrera, donde Hoyt (2005) estableció propuestas muy relevantes para el desarrollo de los estudiantes que, aún hoy, constituyen un reto para los sistemas educativos y donde estudios como los desarrollados por Lee, Rojewski y Hill (2013), Lerchundiet al. (2015), Arnoux et al. (2018), Cohen et al. (2018) entre otros, ponen el énfasis en las aspiraciones académicas, la planificación de los estudios, la toma de decisiones, las influencias externas o la reflexión activa, como elementos definitorios a la hora de atender las necesidades vinculadas a la orientación para la carrera. En cualquier caso, se han visto potenciados los esfuerzos institucionales para tratar de responder a las necesidades de orientación de los estudiantes universitarios, ya sea a través del desarrollo de normativas referidas a los Planes de Acción Tutorial (Pereira et al., 2019), así como a través de la potenciación de otros servicios o documentos universitarios que emergen o se consolidan para complementar la labor del docente, favorecer la adaptación a la vida académica del estudiantado y consolidar nuevas estructuras para dar una respuesta de calidad (Allueva et al., 2016; Ursin, 2017).

En definitiva, estas variables aparecen como determinantes para poder ofrecer, desde la Universidad, un óptimo diagnóstico de las necesidades que presenta el alumnado de nuevo ingreso en relación a su proceso formativo y así, diseñar prácticas asociadas a elementos como los programas de orientación y tutoría desde los que se ofrezca una atención efectiva a esas demandas garantizando una adecuada transición y ajuste al mundo universitario (Bernardo et al., 2017); para ello, como afirman Moreno-Yaguana y Sánchez García (2018) existen diversos postulados que oscilan desde enfoques más sistémicos (modelos de Kaufman o Stufflebeam y Shinkfield) hasta otros más próximos a posiciones clínicas (Pérez-Campanero), pero con independencia de ello sigue siendo muy necesario realizar contribuciones en forma de instrumentos que potencien los recursos existentes para llegar a este fin. El proyecto de investigación educativa que da forma a este trabajo emerge ante cifras como las ofrecidas por el Consejo de Rectores de las Universidades Españolas, en su informe "La Universidad Española en Cifras para el curso 2017/2018", en las que se deja entrever una tasa de abandono del $16,81 \%$ en el primer año de estudios universitarios en las titulaciones de la rama de ciencias sociales y jurídicas (CRUE, 2019). Esta preocupante situación es compartida por los presupuestos del Plan Estrategia 2020 de la Comisión Europea donde se insta a reducir los niveles de abandono escolar a cifras inferiores al $10 \% \mathrm{y}$, por ende, identificar las necesidades se convierte en un reto importante para que la orientación pueda contribuir a tal fin.

\section{Objetivos}

Como objetivo general se propone crear y validar un instrumento que permita indagar en los factores clave para la detección de necesidades de orientación en estudiantes universitarios de nuevo ingreso. De manera particular se establecen dos objetivos específicos: 
a) Desarrollar una fundamentación teórica actualizada y específica desde la que obtener variables asociadas a las necesidades de orientación de los estudiantes universitarios de nuevo ingreso.

b) Realizar un análisis de las propiedades psicométricas del instrumento construido a partir de análisis descriptivos y de correlación de las escalas (identificar factores emergentes) y mediante procedimientos de validación y fiabilidad (valoración de la consistencia interna y el análisis factorial de las escalas).

\section{Método}

Se utiliza una metodología cuantitativa siguiendo un método tipo encuesta con un diseño transversal. El enfoque que centra la investigación es de corte hipotético-deductivo, apoyado en las premisas teóricas aportadas para la elaboración de la estructura del cuestionario y posterior identificación de factores subyacentes a cada una de las escalas: 1) Motivaciones de acceso a los estudios; 2) Necesidades de orientación académica; 3) Necesidades de orientación de carrera o acompañamiento.

Para dar respuesta al objetivo planteado se desarrollan procedimientos de validación de contenido (revisión de literatura y juicios de expertos) y validación empírica (de cada una de las escalas se estudian las siguientes propiedades psicométricas: Correlación Ítem-Total, Fiabilidad, Dimensionalidad y evaluación de ajuste del modelo; por último, se presenta la confirmación factorial de la escala completa).

\section{Muestra}

La población de referencia es la conformada por los estudiantes de nuevo ingreso de las titulaciones del ámbito de la Educación de las Facultades de Educación, Psicología y Ciencias del Deporte de la Universidad de Huelva en el curso 2018/2019 ( $N=702)$. El alumnado ha participado de manera voluntaria, a través de un muestreo de tipo intencional, respetando la presencia de las diferentes titulaciones del ámbito de la Educación adscritas a dicha Facultad, alcanzando una muestra de 376 estudiantes (21\% hombres; 79\% mujeres, con una media de edad de 20,79 años) matriculados en las titulaciones de grado en Educación Infantil $(27,9 \%)$, Educación Primaria $(48,9 \%)$ y Educación Social $(23,1 \%)$, contribuyendo a la representatividad de la muestra y, aumentando así los niveles de confiabilidad y los niveles de sesgo (se asume un error muestral del $5 \%$ para un nivel de confianza del $95 \%$ ). No lo consideramos ideal pero sí razonablemente suficiente para un estudio descriptivo.

Como rasgos definitorios, la mayoría $(90,4 \%)$ cursa por primera vez estudios universitarios y el acceso se produce en gran medida desde bachillerato $(56,1 \%)$, seguido de ciclos formativos de grado superior (39,9\%). Cabe resaltar, por una parte, que solo un $40,2 \%$ ha tenido claro "desde siempre" que la titulación que cursa es la que quería, seguido de un $18 \%$ que asegura que toma la decisión en bachillerato y un $15 \%$ que lo hace justo en el momento del acceso a la Universidad, lo cual se ve reflejado en el alto porcentaje (73,9\%) de estudiantes que afirman que al iniciar los estudios universitarios, su grado de conocimiento sobre la titulación era muy escaso (promedio de 
4,46 en una escala sobre 7 puntos); y por otra parte, se preguntaba por los motivos que le llevaron a elegir la titulación que cursan y, aunque un $78,7 \%$ elige sus titulaciones como primera opción, existen diferencias en las prioridades de esta elección, en concreto, aquellos que la eligen en primera opción lo hacen en un $94,6 \%$ por preferencia o gusto, también por poseer habilidades y aptitudes (72\%), mientras que los que eligen la carrera en segundo o tercer lugar lo hacen mayoritariamente $(91,3 \%)$ por no disponer de plaza en otras titulaciones, y solo el $27 \%$ reconoce tener esas habilidades y aptitudes para la profesión cursada.

\section{Procedimiento}

A continuación, se sintetizan las fases fundamentales del estudio:

Fase 1: validación teórica. Se realizó una revisión de literatura para construir un cuestionario de 58 ítems focalizados en las necesidades de orientación del alumnado universitario y basados en trabajos que profundizan en este marco conceptual como el de Figuera y González (2014) aportando los momentos claves en el proceso de ingreso en la universidad y las variables/necesidades que se asocian a estos $y$, otros trabajos que muestran propiedades psicométricas o comparten instrumentos; de una forma general, Moreno-Yaguana y SánchezGarcía (2018) diseñan y validan un cuestionario con tres factores asociados a necesidades de tipo personal, profesional y académica (en la misma línea que el trabajo de Salmerón et al., 2005) y, de manera más específica, Árias et al. (2005) parten de un cuestionario que atiende a la orientación personal (factor clave en el trabajo de Farriols et al., 2014), las expectativas académicas (diferenciadas en generales y específicas por Sánchez-García, 1998) y profesionales, las dificultades en los primeros momentos o las condiciones para los estudios (Manzano-Soto y Roldan-Morales, 2015, diferencian entre las variables ligadas a los momentos antes de ingresar a la Universidad y al momento durante los estudios); Escolano et al. (2019) identifican las necesidades de tipo geográfica y administrativa; Campoy y Pantoja (2000) también aportan los factores ligados al plano profesional, de carrera, de salud y asistenciales; y finalmente, Hofstadt et al. (2005) identifica necesidades relativas a los planes de estudios, exámenes, estructuras de la Universidad, información sobre la biblioteca, los recursos extrauniversitarios y sobre el docente.

Fase 2: validación de contenido. Tras la revisión de literatura, el cuestionario resultante se sometió a un proceso de validación de contenido mediante el juicio de seis expertos de tres universidades españolas (Universidad de Huelva, Sevilla y La Laguna), seleccionados por su amplia trayectoria y relevancia profesional en el marco de la orientación universitaria (más de 10 años de servicio, con estabilidad en la Universidad y desarrollando líneas de investigación en el marco de la Orientación y la Tutoría) y por el amplio conocimiento en metodología cuantitativa y en análisis psicométricos (profesorado del área de Métodos de Investigación y Diagnóstico en Educación o Didáctica y Organización Escolar, con publicaciones asociadas a la validación de instrumentos de medición/evaluación). A través de una escala tipo Likert con seis categorías $(0=$ nada adecuado y $5=$ totalmente adecuado; acompañada cada categoría con un espacio para las observaciones) se invita a valorar criterios ligados a la claridad (formulación de la pregunta), suficiencia (preguntas suficientes para abordar la dimensión) y pertinencia (relación a las distintas dimensiones). Los cambios sugeridos invitan a: suprimir una categoría destinada a la orientación personal (10 ítems) puesto que los expertos entienden que son apreciaciones difícilmente asumibles por el profesorado universitario; así como reformular tres ítems cuya redacción no poseía una óptima claridad y eliminar 4 ítems cuya pertinencia es valorada negativamente por tres jueces.

Fase 3: pilotaje. Tras la toma de decisiones en el juicio de expertos, se aplica el instrumento a un grupo de 30 estudiantes para realizar una prueba piloto y establecer una aproximación a la fiabilidad del instrumento $(\alpha=.809)$. 
Fase 4: distribución y procesamiento de los datos. Construido el instrumento (Tabla 1) la recogida de información se prolonga durante una semana, una vez avanzado el segundo semestre del primer curso universitario, con el objetivo de acceder a un estudiantado que prácticamente ha finalizado el primer año y ha podido evidenciar diferentes necesidades de orientación en distintos momentos. En este sentido, se informó a los estudiantes acerca de los objetivos y sentido de la investigación que se estaba realizando, se solicitó su participación voluntaria y se garantizó el anonimato de las respuestas obtenidas (se aseguró el derecho a la intimidad, la protección de datos personales, la integridad física y mental, o la no discriminación). Para el tratamiento de los datos, en un primer momento, se ha realizado un análisis descriptivo de los ítems que conforman cada una de las secciones del cuestionario, comprobando así la distribución normal de los datos a través de la asimetría y curtosis, así como la dispersión de los mismos por medio de las medias y desviaciones típicas. Para todas las escalas se hallan valores aceptables en cuanto a la asimetría y curtosis (recomendado \pm 1 ) y suficiente variabilidad en los distintos ítems que las conforman. Con el propósito de explorar la capacidad de la relación entre los ítems que conforman cada escala, se analizan las correlaciones de estos a través del método de Pearson.

En un segundo momento se procede al análisis de la consistencia interna de las tres secciones mediante el empleo del estadístico Alfa de Cronbach, con el objetivo de identificar los ítems menos consistentes y excluir aquellos que harían mejorar la fiabilidad de escala (se utiliza como criterio para la exclusión de los ítems poseer un valor $<0.3$ ). Con la intención de profundizar en el análisis de la estructura de cada escala, cada una de estas se ha sometido a un análisis factorial con método de máxima verosimilitud y rotación Varimax, tratando así de buscar grupos de variables con significado común y considerar sus respectivas dimensionalidades. Una vez determinada la estructura de cada factor se evalúa el ajuste del modelo, recurriendo a un procedimiento de análisis de ecuaciones estructurales mediante el programa AMOS v.18 y empleando como referencia los criterios estipulados por Byrne (2010) y Kline (2010) (CMIN/DF entre 2 y 5, CFI e IFI > .9, RMSEA < .06 y HOELTER > 200). El programa utilizado para el tratamiento estadístico de los datos fue el SPSS 17.0.

\section{Resultados}

A continuación, se presentan los resultados atendiendo al objetivo planteado y a los bloques de contenidos abordados en este estudio que configuran las escalas de las que se compone el instrumento creado.

La estructura final del instrumento denominado "Cuestionario sobre las Necesidades de Orientación en Universitarios/as de Nuevo Ingreso (NOUNI)" (Disponible en Anexo) está formada por 44 ítems agrupados en las siguientes secciones:

1) Datos sociodemográficos y características académicas de ingreso (14 ítems);

2) Motivaciones de acceso a los estudios (10 ítems);

3) Necesidades de orientación académica (10 ítems);

4) Necesidades de orientación de carrera o acompañamiento (10 ítems). 
Las opciones de respuesta para las dos primeras secciones son dicotómicas o politómicas (una sola opción válida), y el resto de las secciones (Tabla 1) se componen de escalas tipo Likert de 7 puntos ( $1=$ nada de acuerdo y $7=$ totalmente de acuerdo).

\section{Tabla 1}

Categorías e indicadores para detectar necesidades de orientación en estudiantes universitarios.

\section{Sección I. Motivaciones de acceso a los estudios}

\begin{tabular}{ll}
\hline Indicadores \\
M1 & Preferencia o gusto por la carrera \\
M2 & Prestigio social \\
M3 & Salidas laborales \\
M4 & Falta de recursos económicos para estudiar otra carrera fuera de tu ciudad/municipio \\
M5 & Consejo de personas allegadas \\
M6 & No conseguir plaza en la titulación que deseaba \\
M7 & Facilidad de los estudios \\
M8 & Duración de los estudios \\
M9 & Poseer un título \\
M10 & Poseer habilidades y aptitudes para esta titulación \\
\hline
\end{tabular}

M10 Poseer habilidades y aptitudes para esta titulación

\begin{tabular}{|c|c|}
\hline \multirow{2}{*}{\multicolumn{2}{|c|}{ on II. Necesidades de orientación académica }} \\
\hline & \\
\hline NA1 & $\begin{array}{l}\text { Considero que el contenido de las asignaturas cursadas se ajusta totalmente a la profesión } \\
\text { docente }\end{array}$ \\
\hline NA2 & $\begin{array}{l}\text { Tengo facilidad para localizar los materiales didácticos necesarios para el estudio de las } \\
\text { asignaturas }\end{array}$ \\
\hline NA3 & nino el volumen de contenido teórico de cada materia \\
\hline NA4 & $\begin{array}{l}\text { e cambiar la forma de estudiar en función del sistema de evalu } \\
\text { ura }\end{array}$ \\
\hline NAt & los criterios de evaluación y calificación de mis asignaturas \\
\hline NA & $\begin{array}{l}\text { Me considero una persona capaz de consolidar grupos de trabajo con el resto de los } \\
\text { compañeros/as }\end{array}$ \\
\hline NA7 & $\begin{array}{l}\text { El profesorado fomenta mi participación en el aula con estrategias activas de enseñanza } \\
\text { (grupos de trabajo, simulaciones, casos prácticos, etc.) }\end{array}$ \\
\hline NA8 & $\begin{array}{l}\text { Me siento capacitado para expresarme y comunicar con claridad (crítica y reflexión) mis ideas } \\
\text { en el aula }\end{array}$ \\
\hline NA9 & la finalidad de las tutorías (desnachn del nrofecor corren ele \\
\hline NA10 & Percibo la existencia de una coordinación entre el profesorado que me ha impartido docencia \\
\hline
\end{tabular}


NC10 Me siento adaptado/a a la vida universitaria (he establecido lazos de amistad con compañeros/as, tengo una relación cordial con el profesorado, etc.)

Fuente: elaboración propia (2020).

Para la validación empírica se analizan las siguientes propiedades psicométricas de cada una de las escalas: Correlación Ítem-Total, Fiabilidad, Dimensionalidad y evaluación de ajuste del modelo.

Escala I "Motivaciones de acceso" (M)

\section{- Correlaciones Ítems-Total}

En las correlaciones ítems-total la mayoría de los ítems correlacionan con el resto significativamente, no obstante los ítems M1 "Preferencia o gusto por la carrera" y M10 "Poseer habilidades y aptitudes para esta titulación" correlacionan menos con el resto, encontrándose además correlaciones significativas negativas; la explicación la podemos encontrar en que aquellos alumnos que han puntuado alto en estos motivos ("gusto por la carrera" y "aptitudes para el desarrollo de la misma") puntúan bajo en el resto de motivos, los cuales no están tan relacionados con la vocación hacia la carrera sino por factores económicos, sociales o por facilidad a la hora de desarrollar los estudios.

\section{- Fiabilidad}

Aplicado el Alfa de Cronbach para estimar la consistencia interna de la escala sobre 10 variables se obtuvo un índice de fiabilidad de .651, valor que se considera algo bajo, aunque aceptable para primeras versiones.

No obstante, examinando los valores parciales asociados a cada uno de los ítems encontramos valores más bajos (comprendidos entre -.158 y .205), lo que nos ha permitido identificar aquellos ítems menos consistentes de cara a la revisión de la escala y su posterior perfeccionamiento (M1, M6 y M10).

Atendiendo a la literatura (valores inferiores a .45) se eliminan los ítems mencionados anteriormente, lo que también es pertinente para el siguiente apartado.

\section{- Dimensionalidad}

Para establecer la estructura factorial se desarrolla un Análisis Factorial Exploratorio (AFE) mediante el análisis de componentes principales como método de extracción Varimax. En la Tabla 2 se representa la estructura factorial exploratoria resultante para los ítems de esta escala.

La extracción inicial dio como resultado dos Factores significativos que explican el $53.38 \%$ de la varianza: un primer Factor que aglutina cuatro ítems que se refieren a motivos de ingreso en la universidad relacionados con la facilidad a la hora de desarrollar los estudios universitarios ("facilidad de los estudios, duración, poseer un título, falta de recursos económicos para estudiar fuera de la universidad") y un segundo Factor formado por aquellos motivos que hacen referencia al estatus social ("prestigio social y salidas laborales"). 


\section{Tabla 2}

Estructura factorial exploratoria de la escala de motivaciones de acceso.

\begin{tabular}{lcr}
\hline \multicolumn{3}{c}{ KMO: 0.749; Bartlett, $\left.X^{2}=451,764 ; \mathrm{gl}=21 ; \mathrm{P}<0.000\right)$} \\
\hline & $F-1$ & $F-2$ \\
M8 &, 757 & \\
M7 &, 754 & \\
M9 &, 681 & \\
M4 &, 603 &, 377 \\
M5 &, 385 &, 847 \\
M3 & &, 768 \\
M2 & & 16,09 \\
VARIANZA EXPLICADA \% 37,28 & \\
Método de extracción: Análisis de componentes principales. \\
Método de rotación: Normalización Varimax con Kaiser. \\
a. La rotación ha convergido en 3 iteraciones. \\
\hline Fuente: elaboración propia (2020).
\end{tabular}

- Estimación de parámetros y evaluación del ajuste

A través del Análisis Factorial Confirmatorio (AFC) se comprueba la adecuación de un modelo de dos factores (Figura 1).

\section{Figura 1}

Estimaciones del modelo para la escala "Motivaciones de acceso".

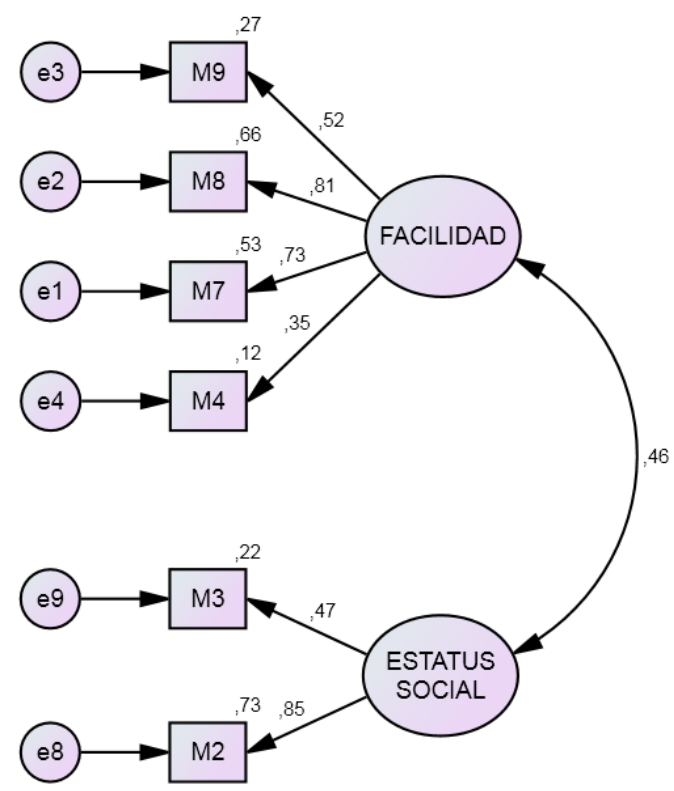

Fuente: elaboración propia (2020). 
En el modelo de medida se aprecian tanto las altas cargas factoriales que conforman cada Factor, siendo todas significativas $(p<.01)$, la elevada correlación existente entre ambos Factores y las elevadas varianzas que son explicadas por el modelo.

Respecto a los índices de ajuste, de forma general, se puede indicar que los resultados reflejaron que el modelo de medida detalla de manera apropiada los datos: la raíz media cuadrática residual, RMSEA =.015 (valor recomendado <.07); el estadístico chi-cuadrado $=1.08$, (valor recomendado entre 2 y 5); IFI (índice de ajuste incremental) $=.99$ siendo el valor recomendado $\geq .90 ; \mathrm{NFI}$ (índice de ajuste normalizado) $=.97$ siendo el valor recomendado próximo a 1; el CFI (índice de ajuste comparativo) $=.99$ siendo el valor recomendado $\geq .90$; HOELTER= 670 (valor recomendado $>200$ ).

Escala II "Necesidades de Orientación Académica" (NA)

\section{- Correlaciones Ítems-Total}

Las correlaciones ítems-total, exceptuando el ítem NA6 "Me considero una persona capaz de consolidar grupos de trabajo con el resto de los compañeros/as", obtienen unos valores que permiten apreciar correlaciones significativas entre todas.

\section{- Fiabilidad}

El Alfa de Cronbach para la escala "Necesidades de Orientación Académica", fue de .792 para 10 elementos. Estos resultados mostraron que la fiabilidad no aumenta si se elimina cualquiera de los ítems, por lo que se decide mantenerlos todos.

\section{- Dimensionalidad}

Atendiendo a la estructura factorial exploratoria resultante del análisis al que ha sido sometido la escala "Necesidades de Orientación Académica" (Tabla 3), esta arrojó un total de tres Factores que explicaron el $58.35 \%$ del total de la varianza.

El primer Factor recoge información sobre aquellos aspectos relacionados con necesidades referidas a contenidos de las asignaturas ("materiales didácticos, contenidos ajustados a la profesión, dominio en el contenido teórico de las materias").

El segundo factor hace referencia a aquellas variables que aluden a una serie de necesidades relacionadas con la "información del profesorado", su "coordinación" y "estrategias de enseñanza".

$\mathrm{Y}$, el tercer factor satura a aquellas variables referidas a las capacidades del alumnado ("cambios en la forma de estudiar, capacidades para consolidar grupos de trabajos", "capacidades para comunicarse y expresar"). 


\section{Tabla 3}

Estructura factorial exploratoria de la escala de necesidades de orientación académica.

\begin{tabular}{lccc}
\hline \multicolumn{4}{c}{ KMO: 0.812; Bartlett, $\left.\mathrm{X}^{2}=847,715 ; \mathrm{gl}=45 ; \mathrm{P}<0.000\right)$} \\
& $F-1$ & $F-2$ & $F-3$ \\
NA2 &, 765 & & \\
NA1 &, 758 & &, 424 \\
NA3 &, 704 & & \\
NA7 & &, 698 & \\
NA10 &, 390 &, 691 &, 817 \\
NA9 & &, 651 &, 531 \\
NA4 & & &, 523 \\
NA6 & &, 485 &, 477 \\
NA8 & &, 458 & 10.51 \\
NA5 &, 357 &, 311 &
\end{tabular}

Método de extracción: Análisis de componentes principales. Método de rotación: Normalización Varimax con Kaiser. a. La rotación ha convergido en 14 iteraciones.

Fuente: elaboración propia (2020)

\section{- Estimación de parámetros y evaluación del ajuste}

Para la confirmación de la estructura factorial resultante en el AFE, se incluyen en el modelo aquellos ítems cuyas cargas factoriales son superiores a >.5, por lo tanto, se decide suprimir de la escala el ítem NA5.

\section{Figura 2}

Estimaciones del modelo para la escala "Necesidades de Orientación Académica".

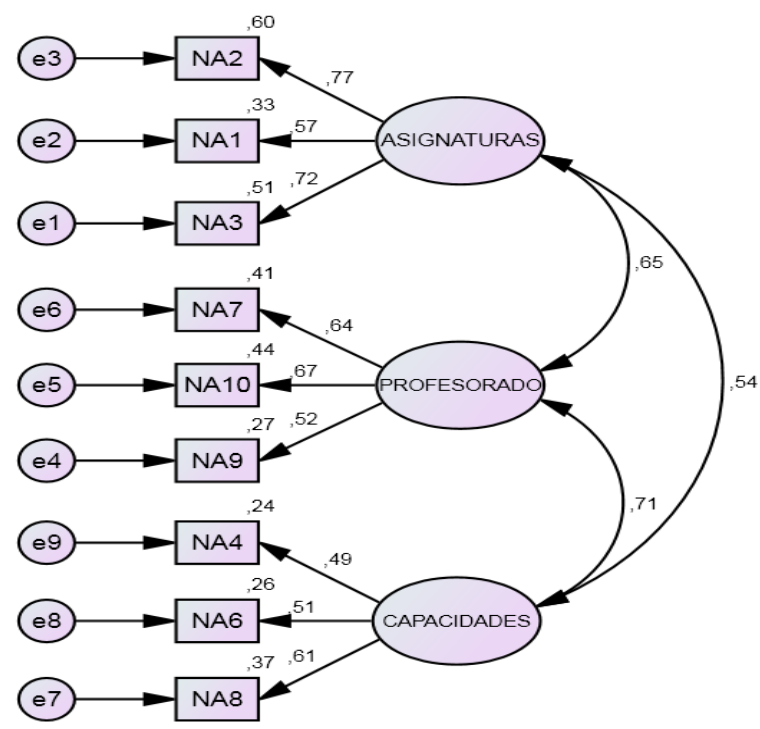

Fuente: elaboración propia (2020). 
Los índices de ajuste del modelo confirmaron una estructura factorial compuesta de tres Factores: RMSEA $=.08$ (valor recomendado <.07); el estadístico chi-cuadrado $=3.38$, (valor recomendado entre 2 y 5 ); IFI (índice de ajuste incremental) $=.91$ siendo el valor recomendado $\geq$ .90; NFI (índice de ajuste normalizado) = .88 siendo el valor recomendado próximo a 1; el CFI (índice de ajuste comparativo) $=.91$ siendo el valor recomendado $\geq .90$; HOELTER= 169 (valor recomendado $>200$ ).

Escala III "Necesidades de Orientación para la Carrera" (NC).

\section{- Correlaciones Ítems-Total}

Las correlaciones ítems-total efectuadas son todas significativas, lo que hace pensar que todos los ítems hacen referencia a una misma dimensión y puntúan en la misma dirección.

\section{- Fiabilidad}

El Alfa de Cronbach para la escala "Necesidades de Orientación para la Carrera", fue de .776 para 10 elementos. Estos resultados mostraron que la fiabilidad no aumenta si se elimina cualquiera de los ítems, por lo que se decide mantenerlos a todos.

\section{- Dimensionalidad}

La estructura factorial resultante del AFE está formada por dos Factores que explican el $47.30 \%$ del total de la varianza (Tabla 4).

\section{Tabla 4}

Estructura factorial exploratoria de la escala de necesidades de orientación para la carrera.

\begin{tabular}{lcr}
\hline \multicolumn{3}{c}{ KMO: 0.813; Bartlett, $\left.X^{2}=770,170 ; g l=45 ; P<0.000\right)$} \\
NC5 & $F-1 \quad$ Componente \\
NC6 &, 708 & \\
NC3 &, 703 & \\
NC4 &, 661 & \\
NC7 &, 654 &, 429 \\
NC8 &, 514 &, 426 \\
NC9 &, 455 &, 771 \\
NC2 &, 437 &, 761 \\
NC1 & &, 731 \\
NC10 & & 13.29 \\
VARIANZA EXPLICADA \% & 34.01 & \\
Método de extracción: Análisis de componentes principales. \\
Método de rotación: Normalización Varimax con Kaiser. \\
a. La rotación ha convergido en 3 iteraciones. \\
\hline Fuente: elaboración propia (2020).
\end{tabular}

El primer Factor describe aquellas necesidades que se relacionan con el asesoramiento en general ("información sobre distintos servicios de la universidad, formación complementaria, asesoramiento en la toma de decisiones, en el plan de estudios, orientaciones ante dificultades"), mientras que el segundo Factor se define a través de variables que aluden a las necesidades de 
adaptación ("los estudios se adaptan a las capacidades de los estudiantes, motivación con la titulación cursada, y adaptación a la vida universitaria, amistades, profesorado, etc.").

\section{- Estimación de parámetros y evaluación del ajuste}

Para la confirmación de la estructura factorial resultante en el AFE se incluyen en el modelo aquellos ítems cuyas cargas factoriales son superiores a $>.5$, por lo tanto, se decide suprimir de la escala los ítems NC8 y NC9.

La estimación de parámetros y evaluación de ajuste del modelo reflejaron la confirmación de la escala "Necesidades de Orientación para la Carrera" en una estructura de dos Factores: RMSEA =.06 (valor recomendado <.07); el estadístico chi-cuadrado= 2.33, (valor recomendado entre 2 y 5 ); IFI (índice de ajuste incremental) = .95 siendo el valor recomendado $\geq .90$; NFI (índice de ajuste normalizado) $=.92$ siendo el valor recomendado próximo a 1; el CFI (índice de ajuste comparativo) $=.95$ siendo el valor recomendado $\geq .90 ;$ HOELTER $=255$ (valor recomendado $>200$ ).

Figura 3

Estimaciones del modelo para la escala "Necesidades de Orientación para la Carrera".

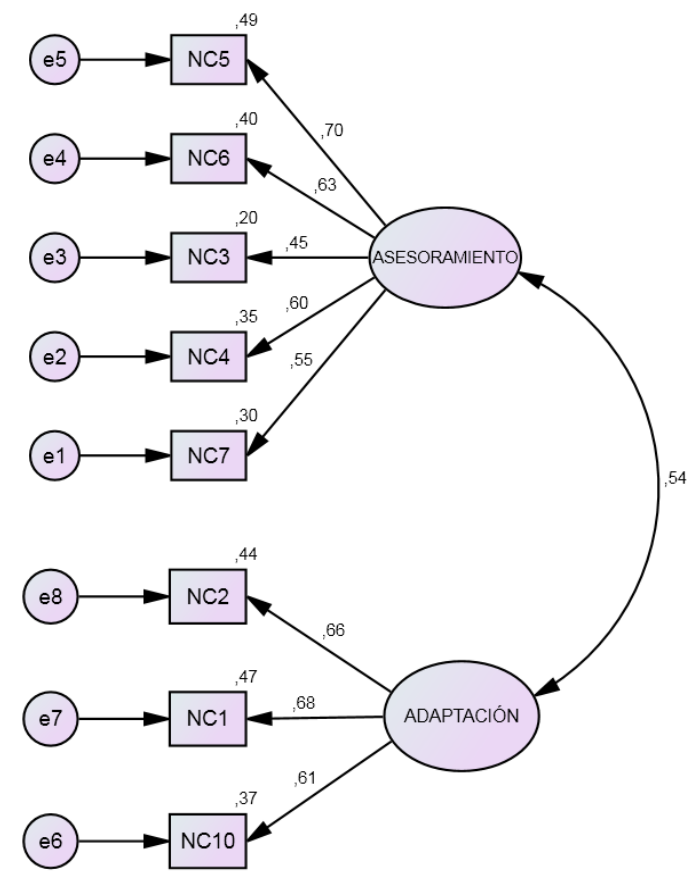

Fuente: elaboración propia (2020).

Confirmación factorial de la escala completa

Como último procedimiento en el proceso de validación del instrumento, la versión final del cuestionario ha sido confirmada por el modelo de ecuaciones estructurales, quedando configurada por 23 ítems distribuidos en siete Factores (Figura 4). A la vista de los resultados obtenidos se puede confirmar la validez del constructo y, además, los altos pesos factoriales y las elevadas 
correlaciones entre la mayoría de los factores muestran un modelo multidimensional válido para la detección de necesidades de orientación en estudiantes universitarios.

\section{Figura 4}

Pesos factoriales del modelo multidimensional.

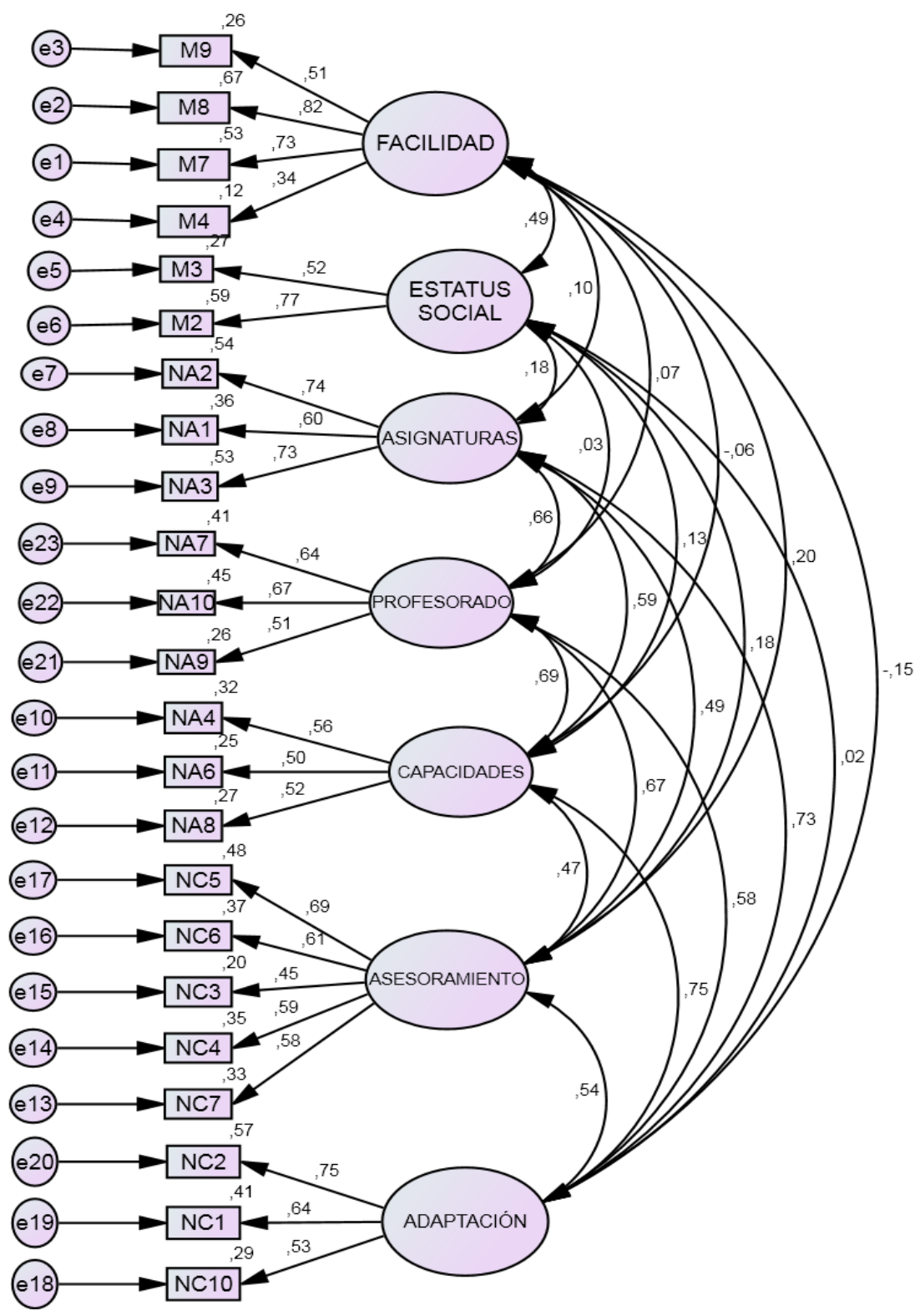

Fuente: elaboración propia (2020).

Así pues, se puede indicar que los índices de ajuste del modelo confirmaron una estructura factorial compuesta de siete Factores (F1-F7): RMSEA $=.052$ (valor recomendado <.07); el estadístico chi-cuadrado= 2.02 (valor recomendado entre 2 y 5); IFI (índice de ajuste incremental) $=.89$ siendo el valor recomendado $\geq .90 ; \mathrm{NFI}$ (índice de ajuste normalizado) $=.81$ siendo el valor recomendado próximo a 1 ; el CFI (índice de ajuste comparativo) $=.89$ siendo el valor recomendado $\geq .90 ;$ HOELTER= 216 (valor recomendado $>200$ ). 


\section{Conclusiones y Discusión}

El estudio desarrollado tenía como objetivo validar un instrumento desde el que indagar en los factores clave para la detección de necesidades de orientación en estudiantes universitarios de nuevo ingreso (NOUNI), contribuyendo así a proporcionar recursos desde los que potenciar la intervención en el área de la orientación universitaria. Al amparo de esta premisa y atendiendo a los resultados obtenidos, el instrumento presentado se puede identificar como válido y fiable. A continuación, esgrimimos los argumentos que han motivado dicha afirmación.

En primer lugar, cabría señalar que en el procedimiento de juicio de expertos se obtuvieron unas valoraciones satisfactorias procedentes de una clara rigurosidad en los juicios emitidos sobre la pertinencia, claridad y suficiencia de los ítems que configuran el instrumento inicial; en el mismo sentido, los resultados derivados del análisis de la consistencia interna de las tres escalas alcanzan valores aceptables-óptimos relativos a la fiabilidad de estas.

En segundo lugar, los procedimientos desarrollados para la validación del constructo teórico dan como resultado un modelo factorial compuesto por siete factores que contribuyen a detectar diferentes tipos de necesidades relacionadas con diversos aspectos de la orientación en la Educación Superior, los cuales sería oportuno considerar cuando se trabaja con el alumnado de nuevo ingreso:

F1 Las motivaciones que abocan a una elección hacia los estudios relacionada con criterios atribuidos a propiedades intrínsecas (objetivas o subjetivas) a los estudiantes (poseer un título universitario, falta de recursos económicos) o a los propios estudios (facilidad, duración).

F2 Las motivaciones que abocan a una elección hacia los estudios relacionada con criterios atribuidos a propiedades extrínsecas (objetivas o subjetivas), ligadas al plano de la consideración social (prestigio social y salidas laborales).

F3 Las propiedades de los elementos curriculares que acompañan a las materias cursadas (materiales didácticos, contenidos ajustados a la profesión, grado de facilidad/dificultad del contenido teórico) y que acaban por otorgarles mayor o menor nivel de aceptación por parte del estudiante.

F4 Las atribuciones que se emiten hacia el rol que adopta el profesorado durante el proceso de enseñanza y aprendizaje (implicación e interés directo por el aprendizaje, el uso de estrategias activas para la enseñanza, o la coordinación entre el equipo docente, o la implicación directa).

F5 Las competencias del estudiante en relación con los resultados de aprendizaje que se esperan de la titulación cursada (dominio de técnicas de estudio, capacidades para consolidar grupos de trabajos, capacidades para comunicarse y expresar).

F6 La información y/o asesoramiento que recibe el estudiante sobre los estudios que va a cursar o que está cursando (Servicios de la universidad, formación complementaria, asesoramiento en la toma de decisiones, características del plan de estudios, orientaciones ante dificultades).

F7 El grado de adaptación que los estudiantes experimentan hacia los distintos elementos vinculados con su estancia en la universidad (la vinculación entre capacidades y 
estudios, el fomento continuado de la motivación hacia la carrera, o la inmersión en la vida universitaria y sus interacciones).

El Análisis Factorial Confirmatorio da como resultado una estructura de modelo multidimensional en la que se identifican los indicadores que realmente constituyen la base de la investigación (necesidades de orientación); esta estructura posee relación con otros trabajos académicos como los de Moreno-Yaguana y Sánchez-García (2018), donde emerge un factor referente a las necesidades de orientación académica en el que se aprecian variables asociadas a nuestros factores F3 y F7; o Manzano-Soto y Roldán-Morales (2015), quienes hablan de necesidades durante la carrera focalizadas en la enseñanza recibida (F3 y F4), los estudios (F6), los aspectos psicosociales (F1,F2,F5 y F7) y, al mismo tiempo, incluyen otras variables que no se incorporan en nuestro instrumento al considerar que no son asumibles por el profesorado (infraestructuras universitarias); por su parte, Escolano et al. (2019) analizan los resultados de un instrumento que sintetiza necesidades de tipo académico (F3, F4, F6), personales y sociales (F5 y F7), y geográficas y administrativas (no comunes a nuestra propuesta); y Hernández et al. (2015) hablan de necesidades académicas (F3, F4, F5, F6) y sociopersonales ( $F 1, F 2, F 7)$ que también acaban por reflejar indicadores compartidos con este trabajo.

Del mismo modo, existen otros trabajos de actualidad enmarcados en la validación de instrumentos para indagar desde la tutoría universitaria en la detección de necesidades de orientación que comparten indicadores con nuestro estudio. Destacamos el trabajo de Amor (2017) que establece cuatro factores (orientación profesional, personal, académica y laboral) de los cuales el dos y el tres agrupan indicadores comunes a nuestros Factores (F5 y F7, F3 y F6 respectivamente); Solaguren-Beascoa y Moreno (2016) obtienen tres factores ligados las áreas de la orientación personal (aspectos de seguridad/confianza), la orientación curricular/extraacadémica (motivaciones e intereses) y orientación académica (aspectos relacionados con las materias) que se relacionan directamente con nuestros factores; Martínez et al. (2019) advierten de la importancia de considerar las necesidades ligadas a la adaptación del contexto universitario (F7), la identidad personal (F1, F2 y F5), la integración (F4 y F7), la enseñanza/aprendizaje (F3 y F6) y finalmente el desarrollo profesional, que queda al margen de nuestra propuesta.

Por último, referenciar que al margen del constructo teórico permanecen varios ítems (M1, M6, M10, NA5, NC8, NC9) que no ayudan a explicarlo; las razones podrían tener relación con la existencia de algunas variables ligadas a lo vocacional que no ayudan a discriminar (los estudiantes que acceden por vocación y con aptitudes, al igual que los que acceden por no conseguir plaza en otra titulación, se entiende que tendrán un menor o mayor número de necesidades, respectivamente, ligadas a los estudios cursados) y también, la breve estancia de los estudiantes en la universidad y por tanto el grado de desconocimiento hacia determinados elementos (criterios de evaluación; distribución teoría-práctica; modalidades de asistencia) se hace patente ante la imposibilidad de contrastar las primeras impresiones.

\section{Limitaciones y Prospectiva}

Las limitaciones se asocian con la distribución del instrumento (posible deseabilidad en las respuestas), su aplicación (un momento concreto en el tiempo no permite desarrollar un pos-test) y la generalización de los datos (sería oportuno corroborar el modelo factorial con muestras más 
plurales); no obstante, estas premisas se convierten en líneas de trabajo para completar el estudio y volver a aplicarlo tras su depuración (poniendo énfasis en mejorar la consistencia interna de la subescala "motivaciones para el acceso a los estudios"). Por otra parte, la limitación en el número de figuras y tablas no ha favorecido la inclusión de todas las evidencias psicométricas logradas en los diferentes procesos de análisis.

Finalmente, destacar la relevancia que cobran actualmente este tipo de trabajos en el seno de la orientación de cara a la identificación de necesidades entre los estudiantes (Creed y Hood, 2015; Moreno-Yaguana y Sánchez-García, 2018; Pereira et al., 2019), atendiendo especialmente a los de nuevo ingreso (Escolano et al., 2019; Hernández et al., 2015), y la posterior intervención por parte del profesorado, generando prácticas de enseñanza-aprendizaje contextualizadas, fortaleciendo la planificación curricular de cada título y contribuyendo a evitar el fracaso y el abandono escolar en el primer año de estudios universitarios. En paralelo, las variables evidenciadas como Factores a tener presente en la detección de necesidades de orientación, también han de ser indicadores positivos tanto para los Servicios de Orientación universitarios, al disponer de material actualizado de manera que sus actuaciones podrán converger junto con las del profesorado (a través de la acción tutorial)y desarrollar iniciativas que promuevan la formación integral del estudiante, como para los Departamentos de Orientación en la etapa de Educación Secundaria, a los que se les brinda un instrumento para identificar posibles necesidades que puedan albergar los estudiantes previo a su acceso a la Universidad y así contribuir a minimizar el impacto de estas en su transición y adaptación a la nueva etapa educativa. De igual modo, este trabajo contribuye a fortalecer una de las líneas de trabajo en el campo de la orientación psicopedagógica relacionada de forma directa con la elaboración de instrumentos con propiedades psicométricas válidas y fiables, de cara a establecer diagnósticos y evaluaciones ajustadas a la realidad de las poblaciones objeto de intervención por parte del orientador o la orientadora.

\section{Referencias bibliográficas}

Álvarez-Pérez, P. R. y López-Aguilar, D. (noviembre, 2016). El profesorado y la valoración de las competencias del alumnado universitario de nuevo ingreso. Trabajo presentado en la International Conference IAEVG. Promoting Equity through Guidance: Reflection Action Impact.

Álvarez-Pérez, P. R. y López-Aguilar, D. (2019). Perfil de ingreso y problemas de adaptación del alumnado universitario según la perspectiva del profesorado. Revista Española de Orientación y Psicopedagogía, 30(3), 46-63. https://doi.org/10.5944/reop.vol.30.num.3.2019

Allueva, P., Zulaica, F. y Abadía, A.R. (2016). Plan de Orientación Universitaria de la Universidad de Zaragoza. Integración de los estudiantes en la Universidad: Binomio Tutor-Mentor. En J. L. Castejón (Coord.), Psicología y Educación: Presente y Futuro (pp. 2273-2281). Asociación Científica de Psicología y Educación.

Amor, M. I. (2017). Análisis de las propiedades psicométricas de una escala sobre las necesidades del alumnado en la tutoría. Revista de Pedagogía, 38(103), 35-57. https://www.redalyc.org/pdf/659/65954978003.pdf 
Árias, M., Álvarez, P. R., García, M.C., Cabrera, J., Martín del Río, M. D. y Robayna, M. (2005). La tutoría como respuesta a las necesidades del alumnado universitario: un estudio en el primer curso de Enfermería de la Universidad de La Laguna. Revista Española de Orientación $\quad y \quad$ Psicopedagogía, 16(2), 319-331. https://doi.org/10.5944/reop.vol.16.num.2.2005.11377

Arnoux, C. et al. (2018). Meaning-Centered Career Intervention for College Students. In V. CohenScali, J. Rossier, y L. Nota (Eds). New perspectives on career counseling and guidance in Europe. (pp. 133-146). Springer.

Artís, M., Suriñach, J., Clar, M. y Duque, J.C. (2008). Estudio de las características individuales que determinan las tasas de abandono en la Universitat de Barcelona. Universitat de Barcelona.

Belloc, F., Maruotti, A. y Petrella, L. (2011). How individual characteristics affect university students drop-out: a semiparametric mixed-effects model for an Italian case study. Journal of Applied Statistics, 38(10), 2225-2239. https://doi.org/10.1080/02664763.2010.545373

Bernardo, A., Cervero, A., Esteban, M., Tuero, E., Solano, P. y Casanova, J. R. (2017). Proyecto E-Orientación, una necesidad desde el campo de la orientación educativa. Revista d'Innovació Docent Universitària, 9, 81-95. https://doi.org/10.1344/RIDU2017.9.7

Byrne, B. (2010). Structural equation modelling with AMOS. Taylor and Francis Group.

Cabrera, L., Bethencourt, J. T., González, M. y Álvarez-Pérez, P. (2006). Un estudio transversal retrospectivo sobre prolongación y abandono de estudios universitarios. Relieve. Revista Electrónica de Investigación y Evaluación Educativa, 12(1), 105-127. http://www.uv.es/RELIEVE/v12n1/RELIEVEv12n1 1.htm

Campoy, T. J. y Pantoja, A. (2000). La orientación en la universidad de Jaén. Revista española de

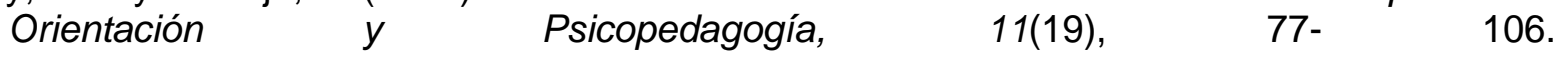
https://doi.org/10.5944/reop.vol.11.num.19.2000.11325

Cohen, V., Rossier, J. y Nota, L. (Eds.). (2018). New perspectives on career counseling and guidance in Europe: Building careers in changing and diverse societies. Zúrich, Switzerland: Springer. https://doi.org/10.1007/978-3-319-61476-2

Conferencia de Rectores de las Universidades Españolas (CRUE) (2019). La universidad española en cifras. Año 2017 y curso académico 2017/2018. http://www.crue.org/SitePages/La-Universidad-Espa\%C3\%B1ola-en-Cifras.aspx

Creed, P. y Hood, M. (2015). The relationship between career adaptability, person and situation variables, and career concerns in Young adults. Journal of Vocational Behavior, 74(2), 210229. https://doi.org/10.1016/j.jvb.2008.12.004

Domingo, J., Fernández-Gálvez, J. y Barrero, B. (2016). El orientador escolar ante el reto de la mejora curricular. Un estudio de caso. Revista Electrónica de Investigación Educativa (REDIE), 18(2), 27-39. https://redie.uabc.mx/redie/article/view/966.

Escolano, E., Lizalde, M., Serrano, R. M. y Casanova, Ó. (2019). Análisis de las necesidades del alumnado de nuevo ingreso en los Grados en Magisterio: Implicaciones para el diseño del Plan de Orientación Universitaria en la Facultad de Educación de la Universidad de Zaragoza. Revista Complutense de Educación, 30(2), 545. https://doi.org/10.5209/RCED.57779

Farriols, N., Vilaregut, A., Palma, C., Dotras, P., Llinares, M. y Dalmau, C. (2014). Estudio descriptivo de un modelo de orientación universitario. Revista Española de Orientación y Psicopedagogía, 25(3), 106- 120. https://doi.org/10.5944/reop.vol.25.num.3.2014.13861 
Fernández-Díaz, M. J. (2006). Necesidades de los estudiantes universitarios ante la realidad del EEES. MEC.

Figuera, P. y González, M. Á. (2014). La intervención orientadora y tutorial en la adaptación y persistencia del alumnado en la universidad. Revista de Orientación Educacional, 28(54), 31-50. https://dialnet.unirioja.es/ejemplar/428127

Figuera, P., Torrado, M., Dorio, I. y Freixa, M. (2015). Trayectorias de persistencia y abandono de estudiantes universitarios no convencionales: implicaciones para la orientación. Revista Electrónica Interuniversitaria de Formación del Profesorado, 18(2), 107-123. http://dx.doi.org/10.6018/reifop.18.2.220101

Harrison, N. (2018). Using the Lens of 'Possible Selves' to Explore Access to Higher Education: A New Conceptual Model for Practice, Policy, and Research. Social Sciences, 7(10), 1-21. https://doi.org/10.3390/socsci7100209

Hernández, C., López, M. y Olmedo, E. (2015). Evaluación de la satisfacción de estudiantes universitarios de nuevo ingreso con el programa E.S.C.L.O.S.A.: experiencia de un programa de orientación. Revista Española de Orientación y Psicopedagogía 26(2), 123135. https://doi.org/10.5944/reop.vol.26.num.2.2015.15219

Hofstadt, C.V., Quiles, Y., Quiles, M.J. y Rodríguez, J. (2005). Necesidades de información al inicio de los estudios universitarios. Educación XX1, (8), 185-199. https://doi.org/10.5944/educxx1.8.1

Hoyt, K.B. (2005). Career Education. History and Future. NCDA.

Íñiguez, T., Elboj, C. y Valero, D. (2016). La Universidad del Espacio Europeo de Educación Superior ante el abandono de los estudios de grado. Causas y propuestas estratégicas de prevención.
Educar,
https://www.raco.cat/index.php/Educar/article/view/311810

285-313.

Kline, R. (2010). Principles and practice of structural equation modelling. The Guilford Press.

Landry, C.C. (2003). Self-efficacy, motivation and outcome expectation correlates of college students, intention certainty (publication No.1254) [Doctoral dissertation, University of Southwestern Louisiana] LSU Doctoral Dissertations. https://digitalcommons.Isu.edu/gradschool dissertations/1254

Lee, I. H., Rojewski, J. W. y Hill, R. B. (2013). Classifying Korean adolescents' career preparedness. International Journal for Educational and Vocational Guidance, 13, 25-45. https://doi.org/10.1007/s10775-012-9236-5

Lerchundi, I., Núñez del Río, M. y González, R. (2015). Career choice in engin students. Anales de Psicología, 31(1), 269-279. https://doi.org/10.6018/analesps.31.1.159161

Llanes, J., Figuera, P. y Torrado, M. (2017). Desarrollo de la empleabilidad y gestión personal de la carrera en Graduados en Pedagogía. Revista Española de Orientación y Psicopedagogía, 28(2), 46-60. https://doi.org/10.5944/reop.vol.28.num.2.2017.20118

Manzano-Soto, N. y Roldan-Morales, C. (2015). Análisis de necesidades de orientación del estudiante de primer año en la Universidad Autónoma de Occidente. Revista Española de Orientación y Psicopedagogía, 26(3), 121-140. https://doi.org/10.5944/reop.vol.26.num.3.2015.16404

Martínez, M. (2009). La orientación y la tutoría en la Universidad en el marco del espacio europeo de Educación Superior (EEES). Revista Fuentes, 9, 78-97. https://revistascientificas.us.es/index.php/fuentes/article/view/2540 
Martínez, P., Pérez, F. J. y González, N. (2019). ¿Qué necesita el alumnado de la tutoría universitaria? Validación de un instrumento de medida a través de un análisis multivariante. Educación XX1, 22(1), 189-213. https://doi.org/10.5944/educxx1.21302

Moreno-Yaguana, P. E. y Sánchez-García, M. F. (2018). Cuestionario de Necesidades de Orientación Universitaria. Revista Iberoamericana de Diagnostico y Evaluación, 47(2), 95109. https://doi.org/10.21865/RIDEP47.2.07

Muñoz, J. L. y Gairín, J. (2013). Orientación y Tutoría durante los estudios universitarios: el Plan de Acción Tutorial. Revista Fuentes, 14, 171-192. https://revistascientificas.us.es/index.php/fuentes/article/view/2358

Pereira, M., Martís R. y Pascual, J. (2019). Evaluación de las necesidades de orientación y educación para la carrera en seis Grados de Educación. Bases para el desarrollo del Plan de Acción Tutorial. Educación XX1, 22(2), 309-334. https://doi.org/10.5944/educxx1.22721

Rodríguez-Moreno, M. L. (2002). Hacia una nueva orientación universitaria. Universidad de Barcelona.

Salmerón, H., Ortiz, L. y Rodríguez, S. (2005). Evaluación de necesidades de orientación universitaria del alumnado marroquí que pretende acceder a universidades españolas. Revista Española de Orientación y Psicopedagogía, 16(2), 225-256. https://doi.org/10.5944/reop.vol.16.num.2.2005.11372

Sánchez-García, M. F. (1998). Las funciones y necesidades de orientación en la Universidad: un estudio comparativo sobre las opiniones de universitarios y profesionales. Revista de Orientación y psicopedagogía, 9(15), 87-107. https://www2.uned.es/reop/pdfs/1998/09-151---087-Sanchez.pdf

Sánchez, Må . F., Manzano, N., Rísquez, A. y Suárez, M. (2011). Evaluación de un modelo de orientación tutorial y mentoría en la Educación Superior a distancia. Revista de Educación, 356, 719-732. https://doi.org/10-4438/1988-592X-RE-2010-356-119

Solaguren-Beascoa, M. y Moreno, L. (2016). Escala de actitudes de los estudiantes universitarios hacia las tutorías académicas. Educación XX1, 19(1), 247-266. https://doi.org/10.5944/educxx1.15586

Suarez Lantarón, B. (2014). Los servicios de orientación profesional en las universidades españolas. Revista de Investigación en Educación, 12(1), 73-91. https://doi.org/10.4995/redu.2013.5565

Urbanaviciute, I., Pociute, B., Kairys, A. y Liniauskaite, A. (2016). Perceived career barriers and vocational outcomes among university undergraduates: Exploring mediation and moderation effects. Journal of Vocational Behavior, 92, 12-21. https://doi.org/10.1016/i.jvb.2015.11.001

Ursin, J. (2017). Tranforming Finnish Higher Education: Institutional Mergers and Conflicting Academic Identities. Revista de Investigación Educativa, 35(2), 307-316. http://dx.doi.org/10.6018/rie.35.2.295831

Vergara, J. R., Boj, E., Barriga, O. y Díaz, C. (2017). Factores explicativos de la deserción de estudiantes de pedagogía. Revista Complutense de Educación, 28(2), 609-630. https://doi.org/10.5209/rev RCED.2017.v28.n2.50009 


\begin{abstract}
Anexo
Cuestionario NOUNI: Necesidades de Orientación en Universitarios/as de Nuevo Ingreso (NOUNI)

Estimado/a estudiante,

El presente cuestionario tiene por objetivo identificar las necesidades de orientación que estás experimentado en tu primer año de carrera, con la finalidad de plantear acciones encaminadas a dar respuesta a tu situación académica personal.

Se trata de una encuesta totalmente anónima, por lo que te solicitamos que seas lo más sincero/a posible en tus respuestas. Encontrarás cuestiones relacionadas con tu situación actual y el perfil de ingreso en la Universidad, otras más próximas a tu proceso formativo (capacidades que posees, propiedades de las asignaturas o el profesorado, etc.) y, finalmente, aquellas que profundizan más en el título que cursas o en un plano institucional.

Gran parte de las respuestas son de opción múltiple (se podrán elegir una o varias opciones) y también tendrás alguna cuestión en la que te pedimos que argumentes tu respuesta para conocer mejor tu opinión.
\end{abstract}

Muchas gracias por tu colaboración.

\title{
Datos sociodemográficos
}

1. Edad:

2. Género: $\square$ Hombre $\mid \square$ Mujer $\mid \square$ Otra categoría

3. ¿Has cursado alguna titulación universitaria anteriormente?

$\square$ Sí, Indica cuál:

$\square$ No

4. Universidad en la que cursas tus estudios:

5. Titulación que cursas actualmente:

$\checkmark$ Grado en Educación Infantil

$\checkmark$ Grado en Educación Primaria

$\checkmark$ Grado en Educación Social

6. Turno en el que estás matriculado/a

$\square$ Mañana $\mid \square$ Tarde $\mid \square$ Tengo asignaturas en ambos turnos

7. Residencia durante el curso

$\square$ En la vivienda familiar habitual

$\square$ En un piso alquilado

$\square$ En casa de algún familiar

$\square$ En colegio mayor o residencia universitaria

$\square$ Otros (especificar):

8. ¿Desarrollas una actividad laboral remunerada (con contrato) actualmente?

$\square$ Sí, en algo relacionado con mis estudios

$\square$ Sí, en algo no relacionado con mis estudios

$\square$ No

9. ¿Has solicitado algún tipo de beca/ayuda para financiar tus estudios en este curso?

$\square$ Sí, Indica cuál/cuáles:

$\square$ No

Perfil de ingreso

10. El acceso a la universidad lo has hecho desde:

$\square$ Bachillerato

REOP. Vol. 32, nำ1, 1er Cuatrimestre, 2021, pp. 92 - 115 [ISSN electrónico: 1989-7448] 
$\square$ Ciclo formativo de Grado Superior

$\square$ Prueba de acceso (Mayores de 25, 40, 45 años)

$\square$ Titulados universitarios

$\square$ Traslado de expediente

$\square$ Otro (especificar):

11. ¿Cuánto tiempo hace que terminaste los estudios que te han habilitado el acceso a la universidad?

$\square$ Menos de 1 año $\mid \square$ Entre 1 y 2 años $\mid \square$ Entre 3 y 4 años $\mid \square$ Más de 5 años

12. Al iniciar los estudios universitarios, tu grado de conocimiento sobre la titulación que cursas era (1 nada; 7 Mucho):

$\square 1$ (nada) $|\square 2| \square 3|\square 4| \square 5|\square 6| \square 7$ (mucho)

13. La titulación universitaria que estás cursando actualmente fue:

$\square$ Tu primera opción

$\square$ Tu segunda opción

$\checkmark$ Tu tercera (o superior) opción

14. Indica en qué medida los siguientes motivos te llevaron a elegir la titulación que cursas (1 nada de acuerdo; 7 totalmente de acuerdo):

\begin{tabular}{|c|c|c|c|c|c|c|c|}
\hline \multirow{3}{*}{$\begin{array}{l} \\
\text { Preferencia o gusto por la carrera } \\
\text { Prestiaio social }\end{array}$} & 1 & 2 & 3 & & & 6 & 7 \\
\hline & & & & & & & \\
\hline & & & & & & & \\
\hline Salidas laborales & & & & & & & \\
\hline $\begin{array}{l}\text { Falta de recursos económicos para estudiar otra carrera } \\
\text { fuera de tu ciudad/municipio }\end{array}$ & & & & & & & \\
\hline Consejo de personas allegadas & & & & & & & \\
\hline No conseguir plaza en la titulación que deseaba & & & & & & & \\
\hline Facilidad de los estudios & & & & & & & \\
\hline Duración de los estudios & & & & & & & \\
\hline Poseer un título & & & & & & & \\
\hline Poseer habilidades y aptitudes para esta titulación & & & & & & & \\
\hline
\end{tabular}

15. ¿En qué momento decidiste cursar los estudios de grado que realizas actualmente?

$\square$ Desde siempre lo he tenido claro

$\square$ Desde la Educación Secundaria Obligatoria (ESO)

$\square$ Desde el Bachillerato

$\square$ Justo en el momento antes de acceder a la universidad

$\checkmark$ Otros (especificar):

Necesidades de orientación académica

Indica en qué medida estás de acuerdo con los siguientes aspectos (1 nada de acuerdo; 7 totalmente de acuerdo)

\begin{tabular}{|l|l|l|l|l|l|l|}
\hline $\begin{array}{l}\text { Considero que el contenido de las asignaturas cursadas se ajusta } \\
\text { totalmente a la profesión docente }\end{array}$ & $\mathbf{2}$ & $\mathbf{3}$ & $\mathbf{4}$ & $\mathbf{5}$ & $\mathbf{6}$ & $\mathbf{7}$ \\
\hline $\begin{array}{l}\text { Tengo facilidad para localizar los materiales didácticos necesarios } \\
\text { para el estudio de las asignaturas }\end{array}$ & & & & & & \\
\hline Domino el volumen de contenido teórico de cada materia & & & & & \\
\hline $\begin{array}{l}\text { Soy capaz de cambiar la forma de estudiar en función del sistema de } \\
\text { evaluación que tenga cada asignatura }\end{array}$ & & & & & \\
\hline $\begin{array}{l}\text { Comprendo los criterios de evaluación y calificación de mis } \\
\text { asignaturas }\end{array}$ & & & & & \\
\hline $\begin{array}{l}\text { Me considero una persona capaz de consolidar grupos de trabajo con } \\
\text { el resto de los compañeros/as }\end{array}$ & & & & & \\
\hline
\end{tabular}

REOP. Vol. 32, nำ1, 1er Cuatrimestre, 2021, pp. 92 - 115 [ISSN electrónico: 1989-7448] 


\begin{tabular}{|l|l|l|l|l|l|l|}
\hline $\begin{array}{l}\text { El profesorado fomenta mi participación en el aula con estrategias } \\
\text { activas de enseñanza (grupos de trabajo, simulaciones, casos } \\
\text { prácticos, etc.) }\end{array}$ & & & & & \\
\hline $\begin{array}{l}\text { Me siento capacitado para expresarme y comunicar con claridad } \\
\text { (crítica y reflexión) mis ideas en el aula }\end{array}$ & & & & & \\
\hline $\begin{array}{l}\text { Conozco la finalidad de las tutorías (despacho del profesor, correo } \\
\text { electrónico, etc.) }\end{array}$ & & & & & \\
\hline $\begin{array}{l}\text { Percibo la existencia de una coordinación entre el profesorado que me } \\
\text { ha impartido docencia }\end{array}$ & & & & \\
\hline
\end{tabular}

Durante tu proceso de aprendizaje en la universidad, ¿has encontrado algún tipo de dificultad?

$\square$ Sí, Indica cuál/cuáles:

$\square$ No

\section{Necesidades de orientación de carrera o acompañamiento}

Indica en qué medida estás de acuerdo con los siguientes aspectos (1 nada de acuerdo; 7 totalmente de acuerdo)

\begin{tabular}{|c|c|c|c|c|c|c|c|}
\hline & 1 & 2 & 3 & 4 & 5 & 6 & 7 \\
\hline Me siento cada día más motivado/a con mi titulación & & & & & & & \\
\hline El nivel de exigencia en los estudios se adapta a mis capacidades & & & & & & & \\
\hline $\begin{array}{l}\text { He tenido asesoramiento en la toma de decisiones (elección } \\
\text { asignaturas, servicios a los que acudir, etc.) }\end{array}$ & & & & & & & \\
\hline $\begin{array}{l}\text { Conozco el Plan de Estudios de mi titulación (peculiaridades de las } \\
\text { asignaturas: número por curso, tipos, créditos, etc.) }\end{array}$ & & & & & & & \\
\hline $\begin{array}{l}\text { He recibido información sobre los distintos Servicios que ofrece esta } \\
\text { Universidad (aulas de informática, salas de trabajo, biblioteca, etc.) }\end{array}$ & & & & & & & \\
\hline $\begin{array}{l}\text { He recibido formación complementaria que me permita completar mis } \\
\text { estudios universitarios (jornadas, cursos, talleres, visitas de } \\
\text { profesionales, etc.) }\end{array}$ & & & & & & & \\
\hline $\begin{array}{l}\text { El profesorado se interesa por mi aprendizaje (da orientaciones ante } \\
\text { dificultades, me escucha cuando lo necesito, etc.) }\end{array}$ & & & & & & & \\
\hline $\begin{array}{l}\text { Los tiempos marcados para compaginar trabajos/actividades prácticas } \\
\text { y estudio del contenido (exámenes) son adecuados }\end{array}$ & & & & & & & \\
\hline $\begin{array}{l}\text { El control de asistencia y la presencialidad me ayuda a implicarme en } \\
\text { la continuidad con los estudios }\end{array}$ & & & & & & & \\
\hline $\begin{array}{l}\text { Me siento adaptado/a a la vida universitaria (he establecido lazos de } \\
\text { amistad con compañeros/as, tengo una relación cordial con el } \\
\text { profesorado, etc.) }\end{array}$ & & & & & & & \\
\hline
\end{tabular}

En resumen, como estudiante universitario/a, ¿qué necesidades de orientación destacarías por orden de relevancia?

$1^{\text {a }}$

$2^{\mathrm{a}}$

$3^{a} \ldots$

Fecha de entrada: 30 marzo 2020

Fecha de revisión: 17 septiembre 2020

Fecha de aceptación: 23 septiembre 2020 